\title{
Antenna system with omnidirectional radiation pattern for systems with phase algorithms of direction finding
}

\author{
Alexander Zhuravlev ${ }^{1, *}$, Alexander Golovkov ${ }^{1}$, Polina Terenteva ${ }^{1}$, Victor Malyshev ${ }^{1}$, \\ Michail Shmyrin ${ }^{2}$, and Nikolay Stenyukov ${ }^{2}$ \\ ${ }^{1}$ Saint Petersburg Electrotechnical University «LETI» 5, Professor Popov Str., 197376, St. Petersburg, \\ Russia \\ ${ }^{2}$ JSC «Scientific-research Institute "Vektor"» 14-a, Academician Pavlova Str., 197376, St. Petersburg, \\ Russia
}

\begin{abstract}
Ommidirectional in azimuth plane antennas with horizontal polarization are used in communication systems of McWILL standard, digital television systems of DVB-T2 standard, radio monitoring systems, semi-active ranging using the target illumination with the television broadcast signal, and many other cases. In many cases, radar and radio monitoring systems use phase methods to determine the azimuth and elevation angle of the target. To view all azimuthal angles, ring arrays consisting of omnidirectional emitters, usually also represented by ring arrays, are used. This paper studies the characteristics of an omnidirectional radiating element of electrically small horizontal dipole elements. An expression is derived that relates the radius of the dipole ring array to the number of dipoles and the variation of the resulting radiation pattern. The results are confirmed by experimental studies.
\end{abstract}

\section{Introduction}

In recent years, great progress has been made [1] in the development and use of semi-active radars for monitoring of unmanned aerial vehicles using the digital TV signal of DVB-T2 standard, McWILL mobile communications systems, etc. for illumination. In all cases, the system operation requires omnidirectional in the azimuth plane antennas with horizontal polarization. When working with vertically polarized signals, a dipole antenna has such radiation pattern, and when working with horizontally polarized signals, an omnidirectional antenna can be obtained with an electrically small horizontal loops. However, electrically small loop antennas have a low efficiency and, consequently, a low gain and vanishingly small operating frequency bands. More promising way is to implement such antennas in the form of a circular array (CA), composed of electrically small antennas with horizontal polarization connected in parallel, for example, dipoles, logoperiodic antennas or Vivaldi emitters placed along the ring with the same angular offset relative to the central axis. As

*Corresponding author: agzhuravlev@stud.eltech.ru 
indicated in the works [2-8], CA of electrically small parallel dipole or slot elements of different shapes are mostly used in such antennas. The antennas presented in these works are designed to operate at frequencies above $1 \mathrm{GHz}$, but when switch to lower frequencies is performed, their dimensions increase rapidly, which limits their use. Relatively simple structures have variation of the radiation pattern of more than $1 \mathrm{~dB}$ [4-5]. The number of elements and the electrical dimensions of the arrays vary greatly in the published papers. At the same time, the number of dipole emitters and the radius of the $\mathrm{CA}$ are the main parameters that determine the good omnidirectional performance and the gain of the antenna. Therefore, the aim of this paper is to study the relationship between the number of dipoles and the radius of the $\mathrm{CA}$ with the variation of the resulting radiation pattern, which will enable designing omnidirectional antennas with horizontal polarization and a given variation of the axisymmetric radiation pattern (RP) in the azimuthal plane.

\section{The simplified mathematical model of CA}

As indicated in the review of omnidirectional antennas [2-8], a uniform radiation pattern with horizontal polarization in the azimuthal plane is obtained by summing the radiation patterns of elementary high-frequency dipoles with identical inphase and equally-amplitude excitation placed along the ring with the same angular offset relative to the central axis. The number of elementary dipoles spread around the circle will obviously be unequivocally related to the radius of the $\mathrm{CA}$ and the size of the dipole (the resonance frequency of the dipole). It allows to establish the relations connecting the electric size of the omnidirectional radiator with variation of the amplitude radiation pattern of the omnidirectional radiator as a whole.

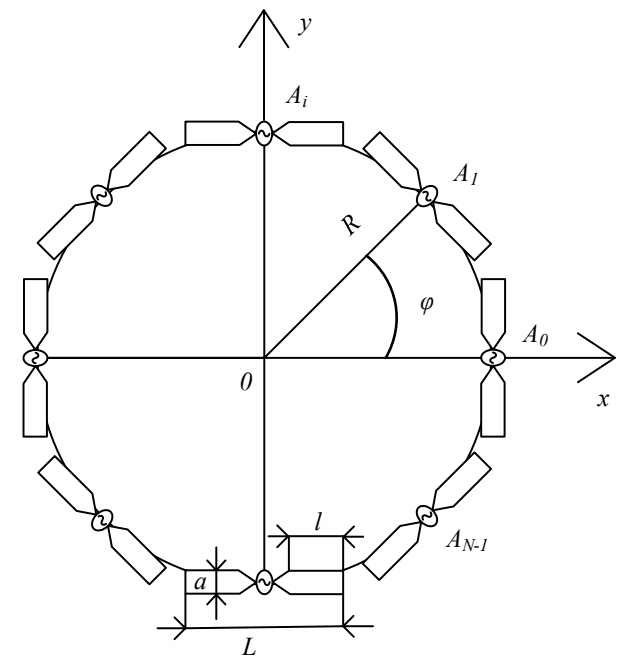

Fig. 1. Circular array model.

Fig. 1 shows a schematic model of the studied omnidirectional radiator. To simplify the analysis of assumptions in this model, the relationship between dipoles will not be taken into account, it will be assumed that dipoles are infinitely thin, the sources that excite them will be connected to the centers of dipoles and the circumference of the omnidirectional emitter will be equal to the product of the number of dipoles by the total length of their arms, i.e. it is assumed that dipoles are located close on the circumference. Under such 
assumptions, the coordinates of the centers of elementary dipoles from element to element vary according to the following expressions (see Fig. 1):

$$
\begin{aligned}
& x_{n}(N, \lambda)=\alpha_{1} \cos \left(\frac{2 \pi n}{N}\right) \\
& y_{n}(N, \lambda)=\alpha_{1} \sin \left(\frac{2 \pi n}{N}\right) ; \alpha_{1}=\frac{R}{\lambda},
\end{aligned}
$$

where $R$ is the radius of the CA, $\lambda$ is the operating wavelength, $n$ is the current dipole number, $n$ varies from 0 to $N-1, N$ is the number of elements.

The radiation pattern of a single $n$ - $t h$ thin dipole antenna, assuming that the current distribution along the dipole conductors is sinusoidal, can be determined by the following expression [9]:

$$
F_{d i p}(\varphi, \lambda, N)=\frac{\cos \left(k l \cos \left(\varphi+\frac{\pi}{2}-\frac{2 \pi n}{N}\right)-\cos (k l)\right)}{\sin \left(\varphi+\frac{\pi}{2}-\frac{2 \pi n}{N}\right)} ; k=\frac{2 \pi}{\lambda},
$$

Where the ratio $\pi / 2-2 \pi n / N$ relates to the rotation of the radiation pattern of the $n$-th dipole antenna when moving from one elementary dipole to another, $l=L / 2-$ the size of the antenna arm.

For a fixed $R$ and $N$ it is necessary to find constraints on the size of the arms of the dipoles $l$, which can be used in the antenna array, as the shape and width of the radiation pattern both depend on these parameters, as well as the input impendance of the dipole. Taking the mentioned above assumptions into account, this constraint will be imposed as:

$$
\frac{\alpha_{2}}{2}=\frac{\pi \alpha_{1}}{N} ; \alpha_{2}=\frac{L}{\lambda}
$$

Using (3) in (2), the expression describing the diagram of the elementary $n$-th dipole element can be converted to the form:

$$
F_{d i p}(\varphi, \lambda, N)=\frac{\cos \left(\frac{2 \pi^{2} \alpha_{1}}{N} \cos \left(\varphi+\frac{\pi}{2}-\frac{2 \pi n}{N}\right)-\cos \left(\frac{2 \pi^{2} \alpha_{1}}{N}\right)\right)}{\sin \left(\varphi+\frac{\pi}{2}-\frac{2 \pi n}{N}\right)}
$$

Taking the interference of waves from the spaced emitters and the amplitude-phase distribution in the dipoles into account, the final expression for the radiation pattern of the CA consisting of the dipoles placed on the ring with the same angular offset relative to the central axis is obtained from the ratio:

$$
\begin{gathered}
F_{\text {array }}(\varphi, N, \lambda)=F_{\text {dip }}(\varphi, N, \lambda) \dot{\mathbf{K}} \\
\dot{\mathbf{K}}=\sum_{n=0}^{N-1} \dot{A}_{n} e^{j k\left(x_{n}(\lambda, N) \sin (\theta) \cos (\varphi)+y_{n}(\lambda, N) \sin (\theta) \sin (\varphi)\right)}
\end{gathered}
$$


where the complex coefficients $\dot{A}_{n}$ take into account the amplitude-phase distribution in the emitters. Under the assumption that all dipoles are inphase and are excited with the same amplitude, we can put them equal to one for all emitters without taking into account their mutual influence.

Using the expressions (4) and (1) in (5), we obtain:

$$
\begin{gathered}
F_{\text {array }}(\theta, \varphi, N, \lambda)=\frac{\cos \left(\frac{2 \pi^{2} \alpha_{1}}{N} \cos \left(\varphi+\frac{\pi}{2}-\frac{2 \pi n}{N}\right)-\cos \left(\frac{2 \pi^{2} \alpha_{1}}{N}\right)\right)}{\sin \left(\varphi+\frac{\pi}{2}-\frac{2 \pi n}{N}\right)} \dot{\mathbf{K}} \\
\dot{\mathbf{K}}=\sum_{n=0}^{N-1} e^{j k\left(\alpha_{1} \lambda \cos \left(\frac{2 \pi n}{N}\right) \sin (\theta) \cos (\varphi)+\alpha_{1} \lambda \sin \left(\frac{2 \pi n}{N}\right) \sin (\theta) \sin (\varphi)\right)}
\end{gathered}
$$

Note that the expression (6) is obtained for vibrator antennas located in the air. An example of the calculation of the radiation pattern of CA using expression (6) for the cases $R / \lambda=0.2$ (a) and $R / \lambda=0.5$ (b) for a different number of dipoles is shown in Fig. 2.
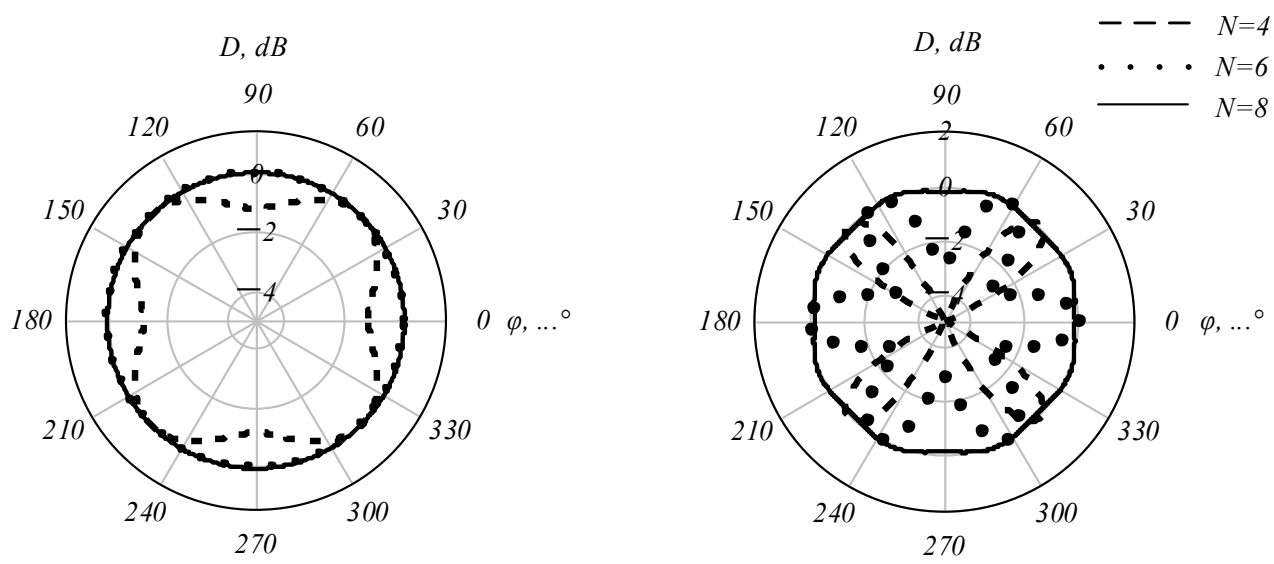

Fig. 2. Radiation pattern of the $\mathrm{CA}$ at various $N$ and $R / \lambda$ ratios.

Multiple calculations of the radiation patterns using the same technique as shown in Fig. 2 allowed to obtain common dependences of the variation of the radiation pattern of CA $(\Delta)$ on the number of dipole elements at different $R / \lambda$ ratios. These dependencies are shown in Fig. $3 a$.
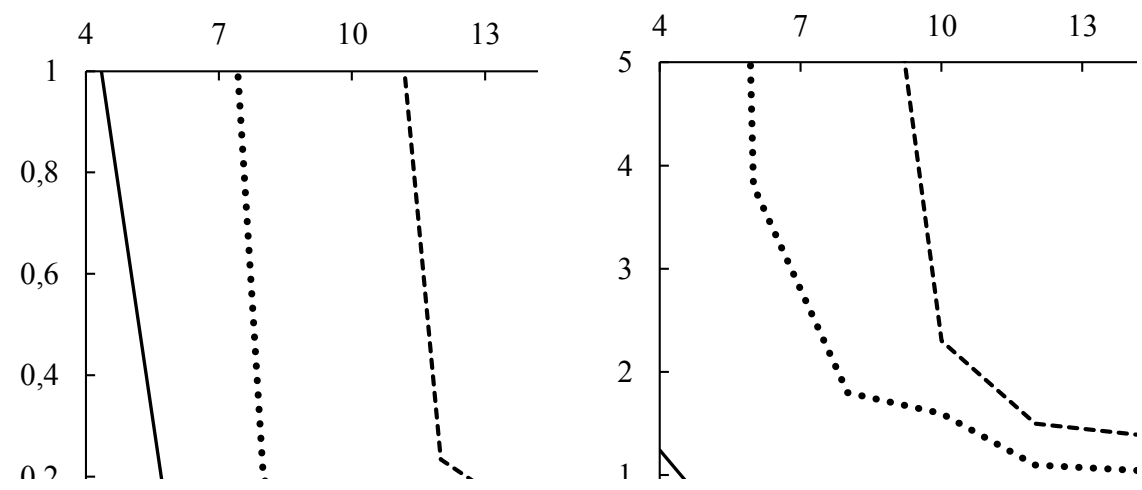
Fig. 3. Variation of the radiation pattern.

In Fig. 3 a solid curve corresponds to $R / \lambda=0.2$; dotted curve - to 0.5 ; dash-dotted curve - to 1 .

To verify the accuracy of the simplified model, electrodynamic modeling of radiation patterns of thin dipoles with the same parameters as in the case of a simplified model was conducted in Ansoft HFSS. The results of the electrodynamic modeling of the variation of the resulting RP are shown in Fig. 3b. Comparison of Fig.3a and Fig.3b shows that not taking the relationship between dipoles into account leads to an increase in the variation of the radiation pattern. With the increase of the electric size of the radius of the CA, the variation of the RP increases faster than in the simplified mathematical model, and with the increase in the number of elements, the variation decreases. Fig. $3 b$ shows that it can be seen that for small $R / \lambda$ ratios, the variation graph is oscillating after $N=8$, reaching a minimum when the number of elements equals $2^{N}$. This is due to the fact that with so many elements, the dipoles are at the vertices of regular geometric shapes. For big $R / \lambda$ ratios this has no effect. Nevertheless, there is a correlation between the data obtained by using a simplified mathematical model and the results of electrodynamic modeling, especially at low frequencies, and due to the curves shown in Fig.3a, the required number of elements and $R / \lambda$ ratio can be selected to ensure a given variation of the radiation pattern.

\section{Optimization of CA using electrodynamic modeling}

Nowadays printed dipole radiators with the arms of a rectangular, triangular or elliptical shape are mainly used in the antenna arrays. The simulation results showed that the least variation of the RP is obtained in the case of using the dipoles with triangular shoulders, whereas dipoles with rectangular arms get the minimum value of $S_{11}$ (such CA will have the greatest broadband). The analysis of structures was carried out in the CST Studio Suite electromagnetic simulation software.

As shown in Fig. 2, the directional patterns of the omnidirectional CA have decrease in the directions of the dipoles. To reduce the variation, it is possible to place printed metal directors in front of dipoles, thereby increasing the gain of dipoles in this direction and as a consequence narrowing their radiation pattern. The simulation results confirmed the effectiveness of this method.

An example of modeling and optimization of one of the CAs with a rectangular shape of dipole arms is shown below. The front view of the radiator is shown in Fig. 4, where the solid line shows the metallization of the upper layer of the dielectric substrate of the FR4 material, and the dash-dotted line shows the bottom layer. The omnidirectional antenna is designed for digital TV range of DVB-T2 standard. According to Fig.3, to obtain a radiation pattern with a variation of not more than $0.2 \mathrm{~dB}$, it uses 8 elementary dipoles with $R / \lambda$ ratio of 0.3 . To simplify the excitation system, a 1:8 power divider is developed, made on the basis of symmetrical two-wire lines with conductors on the upper and lower sides of the dielectric substrate. On both sides in the center of the substrate there are fastening elements which are represented by metal circles connected to the outputs of the balancing transformer. 


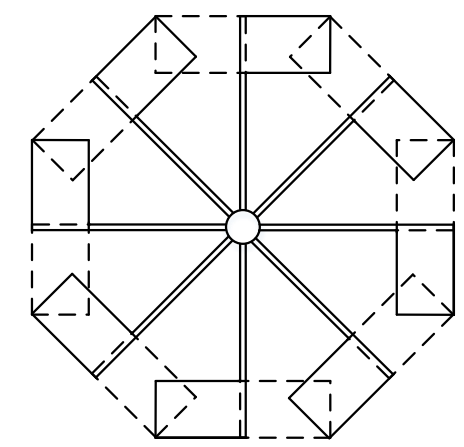

Fig. 4. Antenna element.

The radiation patterns of the simulated CAs in the angular and azimuthal planes are shown in Fig.5a,b respectively, and Fig.5c shows the variation of the phase RP versus the azimuthal angle.
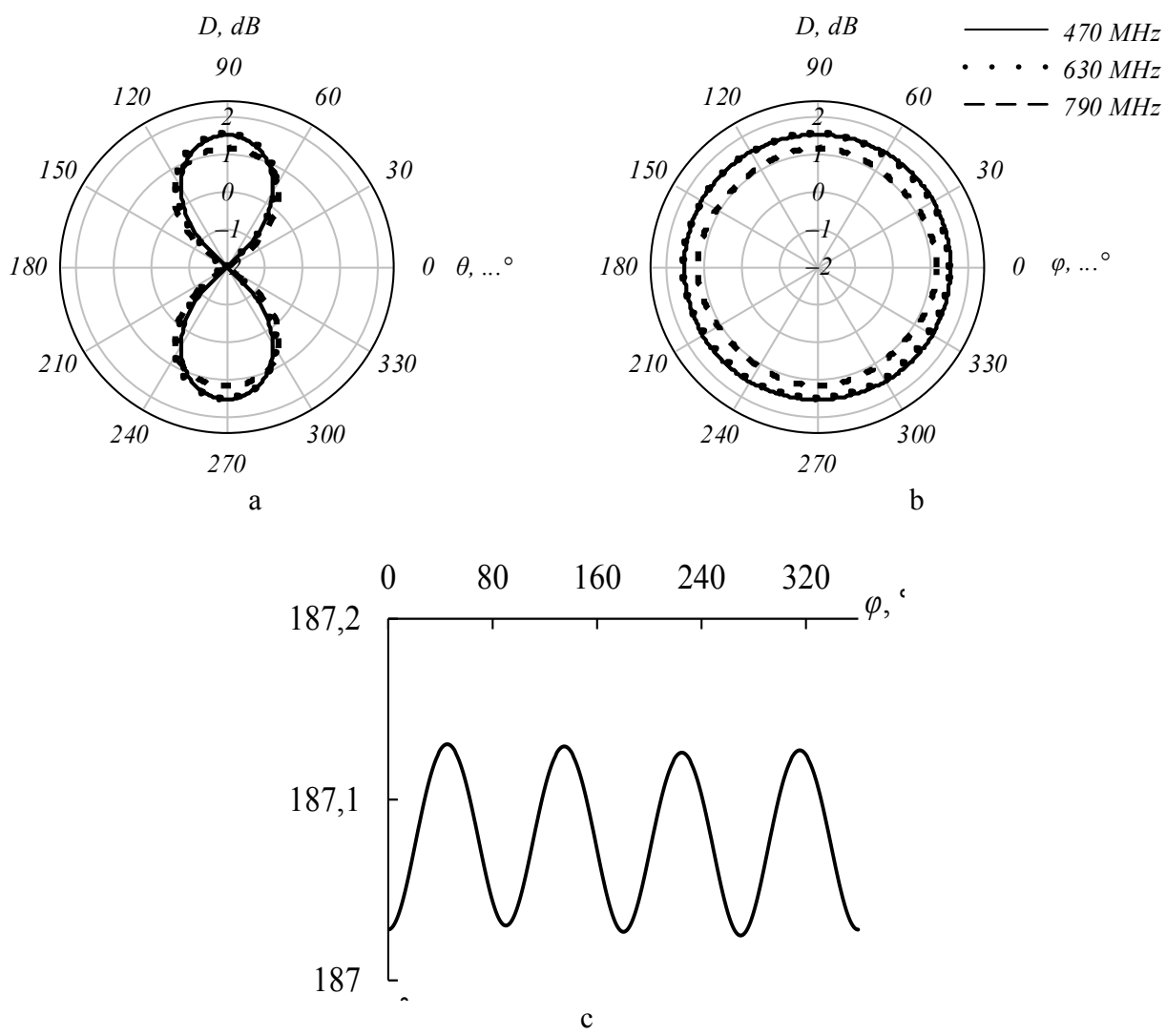

Fig. 5. Characteristics of the antenna system.

\section{Experiment result}

According to the simulation results, a prototype of an omnidirectional CA was made. Dipole elements were made using a three-layer dielectric substrate of FR-4 material with layer's thickness of $3 \mathrm{~mm}$ each. The substrate layers were glued with epoxy adhesive. The arms of printed dipoles and conductors of two-wire lines forming a 1:8 power divider are 
made on the outer sides of the first and third layers of the substrate. The central conductors of the first and third layers are connected to the fingers of a balancing transformer, which is represented by a coaxial $50 \mathrm{Ohm}$ cable with ferrite rings on the outer braid. The layer structure of an antenna is shown in Fig.6A, and Fig.6b illustrates the assembled antenna.

Experimental and calculated characteristics of the modulus of reflection coefficient on the input port of the antenna and the radiation pattern at different frequencies are shown in Fig.7a,b respectively.

As shown in Fig.7a, (the solid curve represents experiment results, the dash-dotted curve represents the modeling) the coincidence of the experimental and calculated S11 curves is quite satisfactory. For Fig. $7 \mathrm{~b}$ solid curve corresponds to the frequency of 470 $\mathrm{MHz}$, dotted curve - to the frequency of $630 \mathrm{MHz}$, dash-dotted curve - to the frequency of $790 \mathrm{MHz}$. Measurement of radiation patterns was performed at the testing area with a bad noise environment. However, the coincidence on both graphs of the theoretical and experimental curves is also satisfactory.

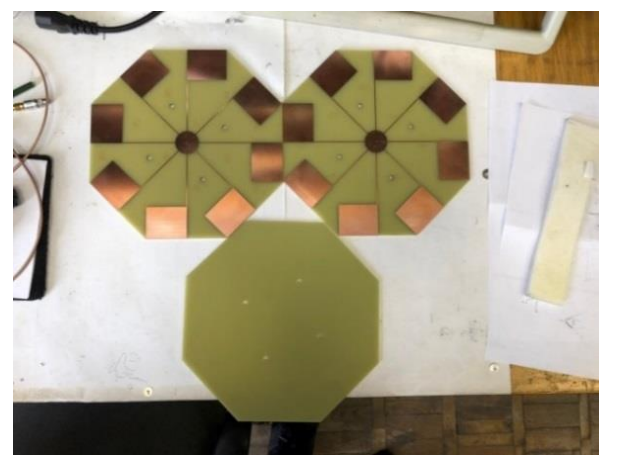

a

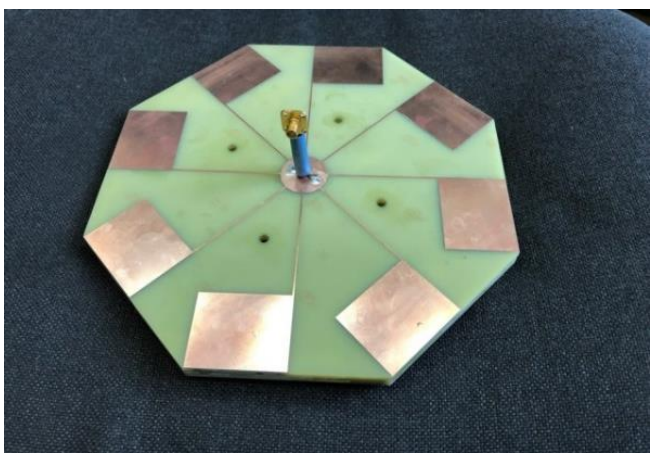

b

Fig. 6. Antenna system.

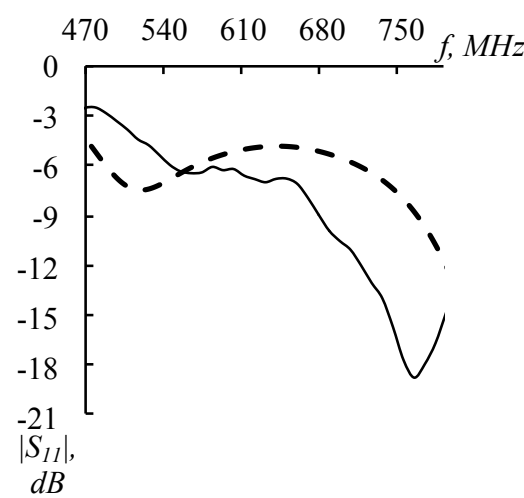

a

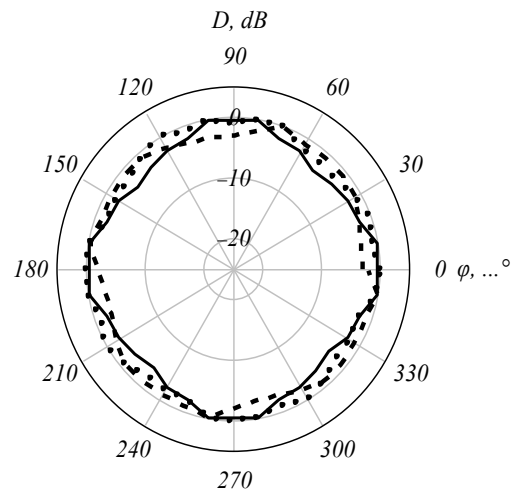

b

Fig. 7. Characteristics of the antenna system.

\section{Conclusion}

The main aspects of designing of omnidirectional antennas in the azimuthal plane based on the CAs made of dipole elements were studied in this paper. The variation of the amplitude 
pattern of less than $0.2 \mathrm{~dB}$ and the phase pattern of less than 1 degree were achieved. These results can be further improved by adding passive metal directors. Currently the authors continue to work on the study of the use of such elements in the antenna arrays.

The work was financially supported by the Ministry of Science and Higher Education of the Russian Federation under the Project ID RFMEF157817X0242.

\section{References}

1. A.V. Barkhatov, V.I. Veremjev, A.A. Golovkov, V.M. Kutuzov, V.N. Malyshev, O.G. Petkau, N.S. Stenjukov, M.S. Shmyrin. Semi-active radar systems for critical facilities monitoring and protection. Journal of the Russian Universities. Radioelectronics., 4, pp. 71-77. In Russ. (2015)

2. D. Punniamoorthy, G. K. Reddy, V. S. Kamadal, G. V. Gopal, K. Poornachary. Design of Patch Antenna with Omni Directional Radiation Pattern for Wireless LAN Applications// Proc. of 2017 Intern. Conf. on Recent Innovations in Signal Processing and Embedded Systems (RISE), 27-29 Oct. 2017, Bhopal, India. Piscataway: IEEE,. pp. 70-74. doi: 10.1109/rise.2017.8378127 (2017)

3. K. Wei, Z. Zhang, Z. Feng. Design of a Wideband Horizontally Polarized Omnidirectional Printed Loop Antenna // IEEE Antennas and Wireless Propagation Letters, (Volume: 11, Issue: 3, January), pp. 49 - 52 DOI:10.1109/LAWP.2012. 2182670 (2012)

4. Z. Wang, Y. Yin, X. Yang, and others. Design of a wideband horizontally polarized omnidirectional antenna with mutual coupling method // IEEE Transactions on Antennas and Propagation, (Volume: 63, Issue: 7, July), pp. 3311 - 3316, DOI: 10.1109/TAP.2015.2429733 (2015)

5. Y. Shi, Z. D. Chen, Y. Yu, and others. A novel omnidirectional horizontally polarized antenna for 4G LTE communications // 2016 11th International Symposium on Antennas, Propagation and EM Theory (ISAPE), Guilin, China, 18-21 Oct., 10.1109/ISAPE.2016.7833879 (2016)

6. H. Zhang, F. Zhang, T. Li, and others. Bandwidth Enhancement of a Horizontally Polarized Omnidirectional Antenna by Adding Parasitic Strips // IEEE Antennas and Wireless Propagation Letters. Vol. 16, pp. 880 - 883, DOI: 10.1109/LAWP.2016. 2613875 (2016)

7. Y. Liu, H. Liu, S. Gong. A Wideband Horizontally Polarized Omnidirectional Antenna Using Tightly Coupled Array Mechanism //Proceeding of 2017 International Workshop on Antenna Technology: Small Antennas, Innovative Structures, and Applications (iWAT), Athens, Greece, 1-3 March, DOI:10.1109/IWAT.2017.7915338 (2017).

8. Wang, S., \& Arai, H. A horizontally polarized notch array antenna with good radiation identity in horizontal plane and its characteristic modes analysis. IEEE Antennas and Propagation Society International Symposium (APSURSI). doi:10.1109/aps.2014. 6904863 (2014)

9. Papakanellos, P. J., Fikioris, G., \& Michalopoulou, A. On the Oscillations Appearing in Numerical Solutions of Solvable and Nonsolvable Integral Equations for Thin-Wire Antennas. IEEE Transactions on Antennas and Propagation, 58, pp. 1635-1644. doi:10.1109/tap.2010.2044319 (2010) 